\title{
Maximum Buckling Load Design of General Cross-section Cylinders Using Lamination Parameters
}

\author{
Ali Khani* ${ }^{\text {,Mostafa M. Abdalla }}{ }^{\dagger}$ and Zafer Gürdal ${ }^{\ddagger}$ \\ Aerospace Structures, Delft University of Technology, Kluyverweg 1, 2629 HS, Delft, The Netherlands
}

\begin{abstract}
For a non-circular cylinder the radius of curvature changes around the circumference. Therefore for constant stiffness non-circular cylinders, some specific locations in the circumference are more prone to buckle. The same problem exists for circular cylinders with non-uniform loading in the cross-section like a cylinder under bending. This explanation brings to the mind the idea of tailoring the material such that the material potential is used more efficiently and if possible all parts of the cylinder contribute in the buckling phenomenon. Since the changes in the radius of curvature and/or loading happens in the circumferential direction, tailoring the material properties in the circumferential direction is a logical pattern. By assigning a certain number of half-waves in the longitudinal direction, the buckling eigen-value problem is solved to find the buckling load and circumferential mode shapes. The inverse of buckling load is approximated using a homogeneous, conservative formulation to increase the computational efficiency during optimisation. This is a hybrid approximation expanded in terms of stiffness linearly and reciprocally. Multimodal optimisation problem is formulated to minimise inverse of critical buckling factor. Variable stiffness design is compared with the quasi-isotropic design.
\end{abstract}

\section{Introduction}

The strong directional properties of fibre reinforced composites can be used more efficiently by taking advantage of fibre placement technology, which allows changing the fibre orientation distribution spatially in a structure. In the general case of shell design, stiffness tailoring would consider the change of local material stiffness (both in plane and bending) over the surface. For a general cross section cylinder like the special case of elliptical one, the radius of curvature changes continuously around the circumference. Therefore for a constant stiffness non-circular cylinder unlike the circular cylinders buckling may occur in some specific locations in the circumference which are weaker. In other words the whole material potential of the cylinder does not contribute in buckling. The same problem exists for circular cylinders with non-uniform loading in the cross-section like a cylinder under bending. This explanation of the buckling problem of non-circular cylinders leads to the idea of tailoring the material such that all points of the cylinder contribute in buckling. Since for a general cylinder the radius of curvature changes only in the circumferential direction, tailoring the material properties in the circumferential direction is a logical pattern. The same concept is valid for circular cylinders when the loading is not uniform in the cross section like a cylinder under bending.

Circumferential tailoring of circular cylinder has been previously studied by Blom et al. ${ }^{1}$ have optimised a circular cylinder to carry maximum buckling load under bending by using constant curvature fiber paths while applying a strength constraint. Analysis and optimisation of the buckling load of elliptical cross-section cylinders under axial compression and torsion has been studied by some researchers ${ }^{2}{ }^{4}$ using fibre angles and laminate thickness as design variables.

In this paper maximum buckling load design of cylinders with general cross-sections under axial loading, bending, and torsion is considered. Lamination parameters which provide a compact representation of the stiffness of a laminate are used as design variables. Use of these parameters reduces the number of design variables regardless of the stacking sequence. In addition, the feasible region of the lamination parameter

*Ph.D. Student, Aerospace Faculty, Kluyverweg 1, Member, AIAA.

$\dagger$ Assistant Professor, Aerospace Faculty, Kluyverweg 1, Member, AIAA.

‡Professor, Aerospace Structures Chair, Aerospace Faculty, Kluyverweg 1, Associate Fellow, AIAA. 
design space is convex..$^{5,6}$ The optimal circumferential distribution of material stiffness is obtained by determining an independent set of lamination parameters at each node in the discretised structure. Variable stiffness designs which have continuously variable lamination parameters over the cross section are introduced and compared with quasi-isotropic design.

\section{Lamination Parameters}

Lamination parameters first presented by Tsai and $\mathrm{Hahn}^{7,8}$ include a compact definition of lay-up configuration. The in-plane and out of plane lamination parameters are specified respectively as

$$
\begin{gathered}
\left(V_{1}, V_{2}, V_{3}, V_{4}\right)=\int_{-1 / 2}^{1 / 2}(\cos 2 \theta(\bar{z}), \sin 2 \theta(\bar{z}), \cos 4 \theta(\bar{z}), \sin 4 \theta(\bar{z})) d \bar{z} \\
\left(W_{1}, W_{2}, W_{3}, W_{4}\right)=12 \int_{-1 / 2}^{1 / 2} \bar{z}^{2}(\cos 2 \theta(\bar{z}), \sin 2 \theta(\bar{z}), \cos 4 \theta(\bar{z}), \sin 4 \theta(\bar{z})) d \bar{z}
\end{gathered}
$$

where $\bar{z}=z / h$ is the normalized through the thickness coordinate $z$ of each layer in the lay-up measured from the middle surface, $h$ is the laminate thickness, and $\theta(\bar{z})$ is the fiber angle at $\bar{z}$. The in-plane stiffness matrix $\mathbf{A}$ and bending stiffness matrix $\mathbf{D}$ can be defined as linear functions of lamination parameters

$$
\begin{gathered}
\mathbf{A}=h\left(\boldsymbol{\Gamma}_{\mathbf{0}}+\boldsymbol{\Gamma}_{\mathbf{1}} V_{1}+\boldsymbol{\Gamma}_{\mathbf{2}} V_{2}+\boldsymbol{\Gamma}_{\mathbf{3}} V_{3}+\boldsymbol{\Gamma}_{\mathbf{4}} V_{4}\right) \\
\mathbf{D}=\frac{h^{3}}{12}\left(\boldsymbol{\Gamma}_{\mathbf{0}}+\boldsymbol{\Gamma}_{\mathbf{1}} W_{1}+\boldsymbol{\Gamma}_{\mathbf{2}} W_{2}+\boldsymbol{\Gamma}_{\mathbf{3}} W_{3}+\boldsymbol{\Gamma}_{\mathbf{4}} W_{4}\right)
\end{gathered}
$$

The lamination parameters cannot be described independently, since the trigonometric terms used in their definition are related. The feasible lamination parameters domain in which these parameters are physically expressive is known for the in-plane ones as

$$
\begin{gathered}
2 V_{1}^{2}\left(1-V_{3}\right)+2 V_{2}^{2}\left(1+V_{3}\right)+V_{3}^{2}+V_{4}^{2}-4 V_{1} V_{2} V_{4} \leq 1 \\
V_{1}^{2}+V_{2}^{2} \leq 1 \\
-1 \leq V_{i} \leq 1(i=1, \ldots, 4)
\end{gathered}
$$

The same feasible region exists also for bending lamination parameters.

The buckling load for a general cylinder depends on both the in-plane stiffness $\mathbf{A}$ and the bending stiffness D. Therefore, definition of the feasible region for the combined set of in-plane and out of plane lamination parameters is needed. In this paper the set of linear constraints extracted by Setoodeh et al. ${ }^{9}$ from the method of successive convex hull approximations is used for the definition of the feasible lamination parameter space.

\section{Buckling Analysis}

Although analytical solution is provided for the buckling of constant stiffness circular cylinders, ${ }^{10}$ it is not applicable in the design of variable stiffness and/or general cross section cylinders since the stiffness and/or section membrane loads are not uniform.

For long cylinders the state of stress under given loading can be approximated by Saint Venant's solution which is only dependent on cross-sectional distribution of stiffness. For buckling analysis of cylinders the mode shape in the longitudinal direction is an integer number of half sinusoidal waves. Hence given the number of axial half waves a finite difference discretisation in the cross-sectional direction is used to find the buckling load and cross-sectional modes by solving the following eigenvalue problem

$$
\left(\mathbf{K}_{t}-\lambda \mathbf{K}_{g}\right) \mathbf{a}=0
$$

where $\mathbf{K}_{t}$ is the global material stiffness matrix, $\mathbf{K}_{g}$ is the global geometric stiffness matrix, $\mathbf{a}$ is the mode shape which contains the deformation degrees of freedom, and $\lambda$ is the load multiplier or buckling factor. Buckling modes are normalised such that

$$
\mathbf{a}^{T} \mathbf{K}_{t} \mathbf{a}=1
$$


Minimum potential energy criterion is applied to formulate the material and geometric stiffness matrix in the buckling eigen-value problem. The Flügge strain-displacement equations which are based on Love's first approximation for general shells are used. ${ }^{11}$

$$
\begin{gathered}
\epsilon_{\alpha}=\frac{1}{A} \frac{\partial u}{\partial \alpha}+\frac{v}{A B} \frac{\partial A}{\partial \beta}+\frac{w}{R_{\alpha}}, \quad \epsilon_{\beta}=\frac{u}{A B} \frac{\partial B}{\partial \alpha}+\frac{1}{B} \frac{\partial v}{\partial \beta}+\frac{w}{R_{\beta}}, \quad \epsilon_{\alpha \beta}=\frac{A}{B} \frac{\partial}{\partial \beta}\left(\frac{u}{A}\right)+\frac{B}{A} \frac{\partial}{\partial \alpha}\left(\frac{v}{B}\right) \\
\kappa_{\alpha}=\frac{1}{A} \frac{\partial \theta_{\alpha}}{\partial \alpha}+\frac{\theta_{\beta}}{A B} \frac{\partial A}{\partial \beta}, \quad \kappa_{\beta}=\frac{\theta_{\alpha}}{A B} \frac{\partial B}{\partial \alpha}+\frac{1}{B} \frac{\partial \theta_{\beta}}{\partial \beta} \\
\tau=\frac{A}{B} \frac{\partial}{\partial \beta}\left(\frac{\theta_{\alpha}}{A}\right)+\frac{B}{A} \frac{\partial}{\partial \alpha}\left(\frac{\theta_{\beta}}{B}\right)+\frac{1}{R_{\alpha}}\left(\frac{1}{B} \frac{\partial u}{\partial \beta}-\frac{v}{A B} \frac{\partial B}{\partial \alpha}\right)+\frac{1}{R_{\beta}}\left(\frac{1}{A} \frac{\partial v}{\partial \alpha}-\frac{v}{A B} \frac{\partial A}{\partial \beta}\right)
\end{gathered}
$$

where $\epsilon_{\alpha}, \epsilon_{\beta}$, and $\epsilon_{\alpha \beta}$ are the normal and shear strains in the middle surface $(z=0)$, also $\kappa_{\alpha}, \kappa_{\beta}$ are the mid surface changes in curvature and $\tau$ is the mid surface twist. Also $R_{\alpha}$ and $R_{\beta}$ are the radii of the $\alpha$ and $\beta$ coordinate curves. Deformations in the $\alpha, \beta$ and $n$ directions are $u, v$ and $w$, respectively.

and

$$
\theta_{\alpha}=\frac{u}{R_{\alpha}}-\frac{1}{A} \frac{\partial w}{\partial \alpha}, \quad \theta_{\beta}=\frac{v}{R_{\beta}}-\frac{1}{B} \frac{\partial w}{\partial \beta}
$$

are the rotations of the normal to the middle surface during deformation about the $\beta$ and $\alpha$ axes respectively. $\alpha$ and $\beta$ coordinate curves for a general shell and $A$ and $B$ as the first fundamental quantities are briefly introduced in Appendix. $n$ is the normal direction to the shell. For a cylinder $\alpha$ is the axial direction while $\beta$ is the circumferential direction $\left(R_{\alpha}=\infty\right)$ and $A$ and $B$ first fundamental quantities are equal to 1 .

A certain number of half-waves for the mode shape in the axial direction is assumed to eliminate the dependency of buckling problem on the axial coordinate $(\alpha)$, therefore the buckling mode shape can be expressed as

$$
\begin{array}{r}
u(\alpha, \beta)=U(\beta) e^{(i K \alpha / L)} \\
v(\alpha, \beta)=V(\beta) e^{(i K \alpha / L)} \\
w(\alpha, \beta)=W(\beta) e^{(i K \alpha / L)}
\end{array}
$$

where $K=m \pi, m$ is the number of axial half-waves and $L$ is the length of the cylinder. Therefore, the buckling problem is only discretised in the cross-sectional direction.

For construction of the potential energy, moderately large rotations are assumed and in order to avoid numerical problems trapezoidal and mid-point numerical integration are used for the membrane and bending part, respectively. By substituting the number of axial half-waves for a general cross-section the straindisplacement relations are written in the matrix form,

$$
\left.\left.\left.\left[\begin{array}{c}
\epsilon_{\alpha} \\
\epsilon_{\beta} \\
\epsilon_{\alpha \beta}
\end{array}\right]_{j}=\mathbf{G}_{m}\right)_{j}\left[\begin{array}{c}
U_{j} \\
V_{j} \\
W_{j} \\
U_{j+1} \\
V_{j+1} \\
W_{j+1}
\end{array}\right], \quad\left[\begin{array}{c}
\kappa_{\alpha} \\
\kappa_{\beta} \\
\tau
\end{array}\right]_{j}=\mathbf{G}_{b}\right)_{j}\left[\begin{array}{c}
U_{j-1} \\
V_{j-1} \\
W_{j-1} \\
U_{j} \\
V_{j} \\
W_{j} \\
U_{j+1} \\
V_{j+1} \\
W_{j+1}
\end{array}\right], \quad\left[\begin{array}{c}
\theta_{\alpha} \\
\theta_{\beta}
\end{array}\right]_{j}=\Omega\right)_{j}\left[\begin{array}{c}
U_{j} \\
V_{j} \\
W_{j} \\
U_{j+1} \\
V_{j+1} \\
W_{j+1}
\end{array}\right]
$$

where

$$
\left.\mathbf{G}_{m}\right)_{j}=\left[\begin{array}{cccccc}
\frac{i K}{2 L} & 0 & 0 & \frac{i K}{2 L} & 0 & 0 \\
0 & \frac{-1}{\Delta} & \frac{1}{2 R_{j}} & 0 & \frac{1}{\Delta} & \frac{1}{2 R_{j+1}} \\
\frac{-1}{\Delta} & \frac{i K}{2 L} & 0 & \frac{1}{\Delta} & \frac{i K}{2 L} & 0
\end{array}\right]
$$




$$
\begin{gathered}
\left.\mathbf{G}_{b}\right)_{j}=\left[\begin{array}{ccccccccc}
0 & 0 & 0 & 0 & 0 & \left(\frac{K}{L}\right)^{2} & 0 & 0 & 0 \\
0 & \frac{-1}{2 \Delta R_{j-1}} & \frac{-1}{\Delta^{2}} & 0 & 0 & \frac{2}{\Delta^{2}} & 0 & \frac{1}{2 \Delta R_{j+1}} & \frac{-1}{\Delta^{2}} \\
0 & 0 & \frac{i K}{\Delta L} & 0 & \frac{2 i K}{R_{j} L} & 0 & 0 & 0 & \frac{i K}{\Delta L}
\end{array}\right] \\
\mathbf{\Omega})_{j}=\left[\begin{array}{ccccccc}
0 & 0 & \frac{-i K}{2 L} & 0 & 0 & \frac{-i K}{2 L} \\
0 & \frac{1}{2 R_{j}} & \frac{1}{\Delta} & 0 & \frac{1}{2 R_{j+1}} & \frac{-1}{\Delta}
\end{array}\right]
\end{gathered}
$$

The constructive elements of the material and geometric stiffness matrix are derived from the minimum potential energy approach,

$$
\begin{gathered}
\left.\left.\left.\mathbf{k}_{m}\right)_{j}=\frac{L \Delta}{2} \mathbf{G}_{m}\right)_{j}^{T}\left(\frac{\mathbf{A}_{j}+\mathbf{A}_{j+1}}{2}\right) \mathbf{G}_{m}\right)_{j} \\
\left.\left.\left.\mathbf{k}_{b}\right)_{j}=\frac{L \Delta}{2} \mathbf{G}_{b}\right)_{j}^{T} \mathbf{D}_{j} \mathbf{G}_{b}\right)_{j} \\
\left.\left.\left.\mathbf{k}_{g}\right)_{j}=-\frac{L \Delta}{2} \boldsymbol{\Omega}\right)_{j}^{T}\left(\frac{\mathbf{N}_{j}+\mathbf{N}_{j+1}}{2}\right) \boldsymbol{\Omega}\right)_{j}
\end{gathered}
$$

$\mathbf{k}_{m}$ and $\mathbf{k}_{b}$ are constructive elements for the membrane and bending parts of the global material stiffness matrix $\left(\mathbf{K}_{t}\right)$ respectively and $\mathbf{k}_{g}$ is the formative element of the global geometric stiffness matrix $\left(\mathbf{K}_{g}\right)$. The global matrices are made by assembling these elements. $\Delta$ is the discretisation interval in the circumferential direction and $\mathbf{N}$ is the vector of membrane forces.

\section{Sensitivity Analysis}

In this section derivation of sensitivity of buckling load with respect to the in-plane and out of plane stiffness of each discretisation point is explained. Material stiffness matrix is explicitly a function of in-plane and out of plane stiffness, while geometric stiffness is a function of load distribution which is subsequently a function of in-plane and out of plane stiffness distribution. Since the conservative approximation in section $\mathrm{V}$ is built for the inverse of buckling load $(r=1 / \lambda)$, the eigen-value problem is reformulated

$$
\left(\mathbf{K}_{g}(\mathbf{N})-r \mathbf{K}_{t}(b)\right) \mathbf{a}=0
$$

where $b$ is an element of either the in-plane or out of plane stiffness matrix. Finding the derivatives of Eq. (19) with respect to stiffness and pre-multiplying the whole expression by $\mathbf{a}^{T}$,

$$
\mathbf{a}^{T}\left(\frac{d \mathbf{K}_{g}}{d b}-r \frac{d \mathbf{K}_{t}}{d b}\right) \mathbf{a}-\frac{d r}{d b}\left(\mathbf{a}^{T} \mathbf{K}_{\mathbf{t}} \mathbf{a}\right)+\mathbf{a}^{T}\left(\mathbf{K}_{g}-r \mathbf{K}_{\mathbf{t}}\right) \frac{d \mathbf{a}}{b}=0
$$

which is simplified to

$$
\frac{d r}{d b}=\mathbf{a}^{T} \frac{d \mathbf{K}_{g}}{d b} \mathbf{a}-r \mathbf{a}^{T} \frac{d \mathbf{K}_{t}}{d b} \mathbf{a}
$$

For the first part of the Eq. (21) the adjoint sensitivity analysis is used, the mode shape is constant $\left(\mathbf{a}=\right.$ cte) and by assigning $g=\mathbf{a}^{T} \mathbf{K}_{g} \mathbf{a}$ we have

$$
\frac{d g}{d b}=\frac{\partial g}{\partial b}+\left(\frac{\partial g}{\partial \mathbf{u}}\right)^{T} \frac{d \mathbf{u}}{b}
$$

In order to calculate $\frac{d \mathbf{u}}{d b}$, derivatives of the equilibrium equation $\mathbf{K}_{s} \mathbf{u}=\mathbf{f}$ are found with respect to the stiffness

$$
\mathbf{K}_{s} \frac{d \mathbf{u}}{d b}+\frac{d \mathbf{K}_{\mathbf{s}}}{d b} \mathbf{u}=0
$$

where $\mathbf{K}_{\mathbf{s}}$ is the static stiffness matrix and $\mathbf{f}$ is the external force vector. The adjoint sensitivity is formulated as

$$
\frac{d g}{d b}=\frac{\partial g}{\partial b}-\mathbf{V}^{T} \frac{d \mathbf{K}_{\mathbf{s}}}{d b} \mathbf{u}
$$


where $\mathbf{V}$ is the adjoint vector evaluated from

$$
\mathbf{K}_{s} \mathbf{V}=\frac{\partial g}{\partial \mathbf{u}}
$$

The second part in the Eq. (21) can be derived easily by finding the derivatives of the constructive elements $\left(\mathbf{k}_{m}\right.$ and $\left.\mathbf{k}_{b}\right)$ of the material stiffness matrix with respect to elements of in-plane or out of plane stiffness.

\section{Conservative Approximation}

Sequential approximation is a method extensively applied in structural optimisation. This technique improves the computational efficiency by avoiding a large number of analysis required to find an optimum. The main goal is to construct separable approximations of objective function and constraints for each discretisation point. Hence one large optimisation problem for the whole structure is replaced by small optimisation problems at each point. The optimisation is implemented on these approximations and after each implementation the design is updated. This procedure is repeated by constructing a new approximation until the solution is converged.

The buckling load is a function of both in-plane and out of plane stiffness. Previously, reciprocal approximation in which the response is approximated in terms of inverse of stiffness properties, has been successfully implemented for compliance and vibration designs. ${ }^{12,13}$ Applying the same method for buckling is not a good idea, since the approximation is found to be non-homogeneous and non-convex. A homogeneous, conservative approximation ${ }^{14}$ is proposed for approximation which is a hybrid formulation of linear and reciprocal approximation. In this approximation the function is expanded using a Taylor series in terms of stiffness matrices and their reciprocals.

The approximation is built for the inverse of buckling load $(r)$ which is a measure of compliance. It is clear from Eq. (21) that the effect of stiffness on $r$ can be subdivided into two parts. The first part which is through the material stiffness matrix is due to local effect of change of stiffness on the buckling load while the load distribution is unchanged. The second part which is from the geometric stiffness matrix is due to the primary effect of change of stiffness on the load distribution which is a global effect. For the approximation, part of $r$ due to the local effect is expanded reciprocally while the part due to the global effect is expanded linearly. The approximation suitable for optimisation is formed after neglecting the constant part of the complete Taylor series at the approximation point and can be expressed as

$$
r \approx \sum_{j=1}^{n}\left(\boldsymbol{\Phi}_{j}^{m}: \mathbf{A}_{j}^{-1}+\boldsymbol{\Phi}_{j}^{b}: \mathbf{D}_{j}^{-1}+\mathbf{\Psi}_{j}^{m}: \mathbf{A}_{j}+\mathbf{\Psi}_{j}^{b}: \mathbf{D}_{j}\right)
$$

where $\boldsymbol{\Phi}$ and $\boldsymbol{\Psi}$ are the sensitivities of inverse of buckling load from the material stiffness matrix with respect to compliance and geometric stiffness matrix with respect to stiffness, respectively. The superscript $m$ and $b$ denotes the sensitivities with respect to the in-plane and out of plane compliance or stiffness, respectively. Subscript $j$ shows the sensitivity with respect to the compliance or stiffness values at point number $j$.

As the applied external load is constant and the internal load redistribution is a function of relative change of in-plane and out of plane stiffness, the summation of global effects from all points at the approximation point is always zero.

$$
\sum_{j=1}^{n}\left(\Psi_{j}^{m}: \mathbf{A}_{j}+\Psi_{j}^{b}: \mathbf{D}_{j}\right)=0
$$

Since the stiffness matrices are always positive definite, Eq.( 27) clarifies that the sensitivity matrix is not essentially positive definite. Therefore convexity of the problem is not promised using reciprocal expansion of the terms related to the global effect. But it is assured at least that the approximation is not concave when these terms are expanded linearly. The local part which is expanded reciprocally is always convex and in this way the whole approximation is guaranteed to be convex.

\section{Multi-modal Design}

For maximising the buckling load, all critical modes should be incorporated in optimisation procedure. Therefore the problem of buckling optimisation is expressed as minimisation of the critical value for inverse 
of buckling load

$$
\min \left(\max \left(r_{i}\right)\right)
$$

where $r_{i}$ (for $i=1,2, \ldots, l$ ) is the value of inverse of buckling load for mode number $i$.

This optimisation problem can be solved using the bound formulation proposed by Olhoff $^{15}$

$$
\min \beta \quad \text { subject to } \quad r_{i} \leq \beta
$$

The problem can subsequently be solved using the dual-method, ${ }^{16}$ which leads to the following optimisation problem

$$
\max _{\mu} \min _{V, W} \sum_{i=1}^{l}\left(\mu_{i} r_{i}\right) \quad \text { subject to } \quad \sum_{i=1}^{l} \mu_{i}=1 \quad \text { and } \quad \mu_{i}>0
$$

where $\mu_{i}$ are the Lagrange multipliers and are determined by maximising the complimentary Lagrangian subject to non-negativity of the Lagrange multiplier, $\mu_{i}>0$, as well as their sum being unity. Therefore, the approximation for the objective function is expressed as

$$
\sum_{i=1}^{l}\left(\mu_{i} r_{i}\right)=\sum_{j=1}^{n}\left(\mathbf{\Phi}_{j}^{m}: \mathbf{A}_{j}^{-1}+\boldsymbol{\Phi}_{j}^{b}: \mathbf{D}_{j}^{-1}+\mathbf{\Psi}_{j}^{m}: \mathbf{A}_{j}+\mathbf{\Psi}_{j}^{b}: \mathbf{D}_{j}\right)
$$

where

$$
\mathbf{\Phi}_{j}^{m}=\sum_{i=1}^{l} \mu_{i}\left(\boldsymbol{\Phi}_{i, j}^{m}\right), \quad \boldsymbol{\Phi}_{j}^{b}=\sum_{i=1}^{l} \mu_{i}\left(\boldsymbol{\Phi}_{i, j}^{b}\right), \quad \mathbf{\Psi}_{j}^{m}=\sum_{i=1}^{l} \mu_{i}\left(\mathbf{\Psi}_{i, j}^{m}\right), \quad \boldsymbol{\Psi}_{j}^{b}=\sum_{i=1}^{l} \mu_{i}\left(\mathbf{\Psi}_{i, j}^{b}\right)
$$

where $l$ is the number of modes and $n$ is the number of nodes. Eq. (30) is separable and can be solved locally at each discretisation point. In other words, the objective function can be minimised by optimising the following local function

$$
\mathbf{\Phi}_{j}^{m}: \mathbf{A}_{j}^{-1}+\boldsymbol{\Phi}_{j}^{b}: \mathbf{D}_{j}^{-1}+\boldsymbol{\Psi}_{j}^{m}: \mathbf{A}_{j}+\mathbf{\Psi}_{j}^{b}: \mathbf{D}_{j}
$$

subject to the constraints on the lamination parameters. ${ }^{9}$

\section{Results}

As three examples the buckling load maximisation of a circular cylinder under bending, an elliptical cylinder under axial compression and also an elliptical cylinder under torsion which have been previously studied in literature are investigated. For multi-modal optimisation, up to 10 axial half-waves are examined and the 10 most critical buckling loads are found and optimisation is implemented on these selected modes. The geometric and material properties of these cylinders are summerised in tables 1 and 2. The azimuth angle in figures starts from the top crown of the cylinder.

The circular cylinder under bending has the same properties as those mentioned by Blom et al., ${ }^{1}$ the objective is to maximise the buckling load with the constant ply thickness. For the mesh convergence study up to 128 discretisation points around the circumference are examined and for the optimisation purposes 64 points are chosen. Around $42 \%$ improvement in the buckling load is achieved with respect to the quasiisotropic design and the buckling load is 7598 in-kips for variable stiiffness design. Blom et al. ${ }^{1}$ have considered constraints on strength, deflection effective stiffness and in-plane fibre curvature and they have reached the buckling load of 6188 in-kips which is $17 \%$ improvement with respect to a baseline stacking sequence composed of $0, \pm 45$ and 90 degree plies. The axial force and strain distribution around the circumference is shown in figure 1. It is clear from this figure that the axial strain distribution around the circumference of the variable stiffness cylinder has a sinusoidal pattern since the two ends of the cylinder remain planar. The strains have greater values in the compression side due to shift in neutral axis towards the tension side. The maximum axial section force on the compression side of the variable stiffness cylinder is less than the maximum axial force on the tension side and the pattern is not anymore sinusoidal. This mechanism which increases the buckling load is due to the tailoring that reduces the local stiffness in the 
compression side and increases that in the tension side. Therefore the load is realeased in the compression side and more tolerated by the tension side. Circumferential critical buckling mode shape is shown in figure 2 .

The elliptical cylinder under axial compression has the properties of the small cylinder studied by Sun and Hyer. ${ }^{2}$ The difference is the nonlinear prebuckling analysis in their work while the analysis in this paper is completely linear. Around 55\% improvement is gained with respect to the quasi-isotropic design. figure 3 shows the critical mode shapes for the quasi-isotropic and variable stiffness cylinder. It can be seen that for quasi-isotropic designs the sections with larger radius of curvature are more prone to buckle. Distribution of axial force is shown in figure 4 while axial strain is uniform. For variable stiffness design the load is distributed such that the sections with larger radius of curvature impart less load and sections with smaller radius of curvature have larger axial force values. In this way the whole parts of the cylinder contribute in buckling phenomenon and buckling load is maximaised. Further comaprisons with the results from Sun and Hyer $^{2}$ will be made based on nonlinear prebuckling analysis in future work.

The elliptical cylinder under torsion has the properties of the small cylinder investigated by Haynie and Hyer. ${ }^{4}$ The buckling torque is improved around $50 \%$ for the variable stiffness design with respect to the quasi-isotropic cylinder. The shear load is the same for variable stifness and quasi-isotropic design, this is while the shear strain is larger for the variable stiffness design and the ratio with respect to the value for quasi-isotropic design is close to the value of improvement in buckling load. In other words, in variable stiffness design the cylinder becomes less stiff, allowing to have larger rate of twist and strain and hence larger buckling torque. Reduced stiffness keeps the shear load almost unchanged. Critical mode shapes are shown in figure 5 .

Final distribution of lamination parameter should be used to retrieve the fibre angles in order to have a practical design. There is a recently developed method for retrieving the fibre angles ${ }^{17}$ which will be used in future work. It is worth to mention that usually a loss of performance happens using the retrieved fibre angles.

Table 1. Geometry of the cylinders

\begin{tabular}{|c|c|c|c|}
\hline Cylinder & Circular $^{1}$ & Elliptical $I^{2}$ & Elliptical $I I^{4}$ \\
\hline$a$ (Semi-major axis) & $24[\mathrm{in}]$ & $0.1250[\mathrm{~m}]$ & $0.1270[\mathrm{~m}]$ \\
\hline$b$ (Semi-minor axis) & $24[\mathrm{in}]$ & $0.875[\mathrm{~m}]$ & $0.889[\mathrm{~m}]$ \\
\hline$L$ (Cylinder length) & $32[\mathrm{in}]$ & $0.320[\mathrm{~m}]$ & $0.292[\mathrm{~m}]$ \\
\hline$H$ (Layup thickness) & $0.1728[\mathrm{in}]$ & $1.120[\mathrm{~m}]$ & $1.118[\mathrm{~m}]$ \\
\hline
\end{tabular}

Table 2. Properties of materials used in cylinders

\begin{tabular}{|c|c|c|c|}
\hline Cylinder & Circular $^{1}$ & Elliptical $I^{2}$ & Elliptical $I I^{4}$ \\
\hline$E_{1}$ & $18.83 e 6[\mathrm{psi}]$ & $130.0[\mathrm{GPa}]$ & $130.0[\mathrm{GPa}]$ \\
\hline$E_{2}$ & $1.317 e 6[\mathrm{psi}]$ & $9.70[\mathrm{GPa}]$ & $9.70[\mathrm{GPa}]$ \\
\hline$G_{12}$ & $7.672 e 5[\mathrm{psi}]$ & $5.00[\mathrm{GPa}]$ & $5.00[\mathrm{GPa}]$ \\
\hline$\nu_{12}$ & $0.32[-]$ & $0.30[-]$ & $0.30[-]$ \\
\hline
\end{tabular}

\section{Conclusion}

Linear buckling analysis of general cross-sectional cylinders is formulated. A conservative hybrid approximation is constructed for the inverse of buckling load. The optimisation is formulated as minimisation of the maximum inverse of buckling load and is solved using bound formulation. Optimisation is performed for buckling under axial compression, bending and torsion and the variable stiffness design is compared with the quasi-isotropic design. By looking at the cross-sectional load distribution of the final design it is tried to understand the mechanisms involved in buckling improvements. Retrieving the fibre angle from lamination parameter distribution will be considered in future work. 


\section{Appendix}

The deformation of a thin shell is fully described by the displacements of its middle surface. In the general theory of surfaces, the equation of the undeformed mid surface is expressed in terms of two independent surface parameters $\alpha$ and $\beta$, whereas each point on the surface is defined by the position vector

$$
\vec{r}=\vec{r}(\alpha, \beta)
$$

The curves on the surface on which the parameter $\alpha$ is kept at a fixed value while $\beta$ changes are called $\beta$ curves, and $\alpha$ curves are described in the analogous manner. There is a one to one correspondence between each $(\alpha, \beta)$ as a point on a definite region and each point on the surface. The tangent vectors to the $\alpha$ and $\beta$ curves are obtained as bellow

$$
\vec{r}_{, \alpha}=\frac{\partial \vec{r}}{\partial \vec{\alpha}}, \quad \vec{r}_{\beta}=\frac{\partial \vec{r}}{\partial \vec{\beta}}
$$

The length of these vectors are denoted by

$$
\left|\vec{r}_{\alpha}\right|=A, \quad\left|\vec{r}_{\beta}\right|=B
$$

The angle between the coordinate curves $\chi$ is calculated as

$$
\frac{\vec{r}, \alpha}{A} \cdot \frac{\vec{r}, \beta}{B}=\cos \chi
$$

Terms $A^{2}, B^{2}$ and $A B \cos \chi$ are called the first fundamental quantities. ${ }^{11}$

\section{References}

\footnotetext{
${ }^{1}$ Blom, A.W., Stickler, P.B., and Gürdal Z. Optimization of a composite cylinder under bending by tailoring stiffness properties in circumferential direction. Composites Part B: Engineering, 2009.

${ }^{2}$ Sun, M., and Hyer M.W. Use of Material Tailoring to Improve Buckling Capacity of Elliptical Composite Cylinders. AIAA journal 46, no. 32008.

${ }^{3}$ Paschero, M., and Hyer M.W. Improvement of Axial Load Capacity of Elliptical Cylindrical Shells. AIAA journal 47, no. $1,2009$.

${ }^{4}$ Haynie, W., and Hyer M.W. Behavior of Elliptical Composite Cylinders in Torsion. 47th AIAA/ASME/ASCE/AHS/ASC Structures, Structural Dynamics, and Materials Conference, 1-4 May 2006, Newport, Rhode Island.

${ }^{5}$ Fukunaga, H., Sekine, H. and Sato M. Optimal design of symmetric laminated plates for fundamental frequency. Journal of Sound and Vibration 171, no. 2, 1994: 219-229.

${ }^{6}$ Foldager, J., Hansen J.S., and Olhoff N. A general approach forcing convexity of ply angle optimization in composite laminates. Structural and Multidisciplinary Optimization 16, no. 2, 1998: 201-211.

${ }^{7}$ Tsai, S.W., Pagano, N.J. "Invariant properties of composite materials." Composite materials workshop. 1968: 62-71.

${ }^{8}$ Tsai, S.W., Hahn, H.T. "Introduction to composite materials." CRC, 1980.

${ }^{9}$ Setoodeh, S., Abdalla, M.M., Gürdal Z. "Approximate Feasible Regions for Lamination Parameters." 11th AIAA/ISSMO Multidisciplinary Analysis and Optimization Conference, Portsmouth, Virginia. 2006.

${ }^{10}$ Brush D.O., Almorth B.O., "Buckling of bars, plates and shells", McGraw-Hill, New York, 1975.

${ }^{11}$ Leissa, A.W. Vibration of shells (NASA SP-288). US Government Printing Office, Washington DC, $1973: 428$.

${ }^{12}$ Setoodeh, S., Abdalla M.M., and Gürdal Z. Design of variable-stiffness laminates using lamination parameters. Composites Part B: Engineering 37, no. 4, 2006: 301-309.

${ }^{13}$ Abdalla, M.M., Setoodeh S., and Gürdal Z. Design of variable stiffness composite panels for maximum fundamental frequency using lamination parameters. Composite structures 81, no. 2, 2007: 283-291.

${ }^{14}$ Haftka, R.T., and Gürdal Z. "Elements of structural optimization." Springer, 1992.

${ }^{15}$ Olhoff, N. Multicriterion structural optimization via bound formulation and mathematical programming. Structural and Multidisciplinary Optimization 1, no. 1, 1989: 11-17.

${ }^{16}$ Fleury, C., and Schmit L.A. "Dual methods and approximation concepts in structural synthesis." National Aeronautics and Space Administration, Scientific and Technical Information Branch, 1980.

${ }^{17}$ IJsselmuiden S.T., Abdalla M.M., Pilaka V.K.R., Gürdal Z. "Design of Variable Stiffness Composite Structures for Advanced Fiber Placement Technology.' SAMPE Symposium and Exhibition, Seattle. 2010.
} 


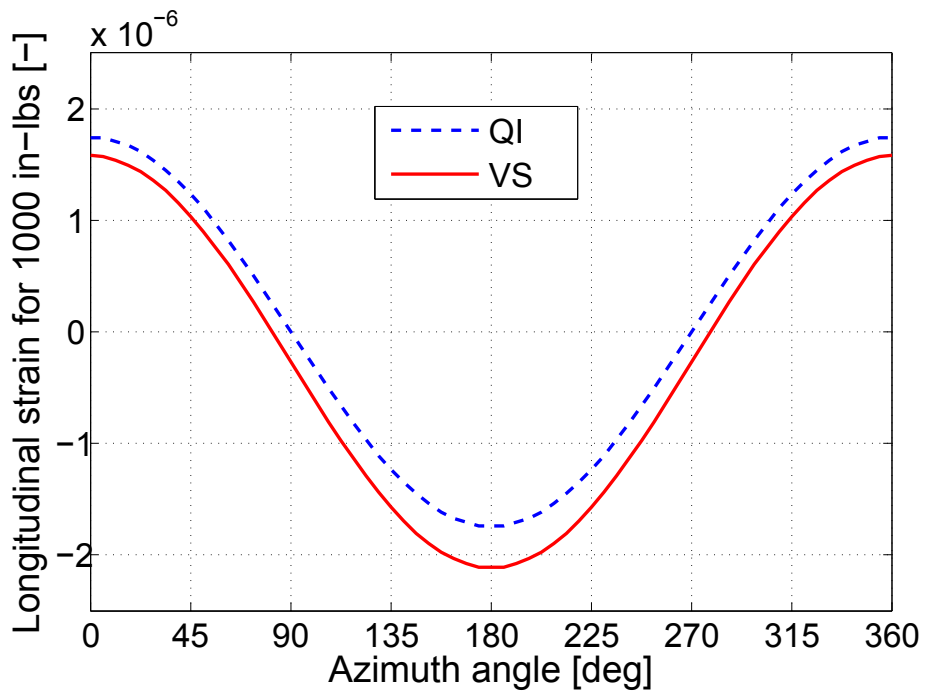

(a) Longitudinal strain

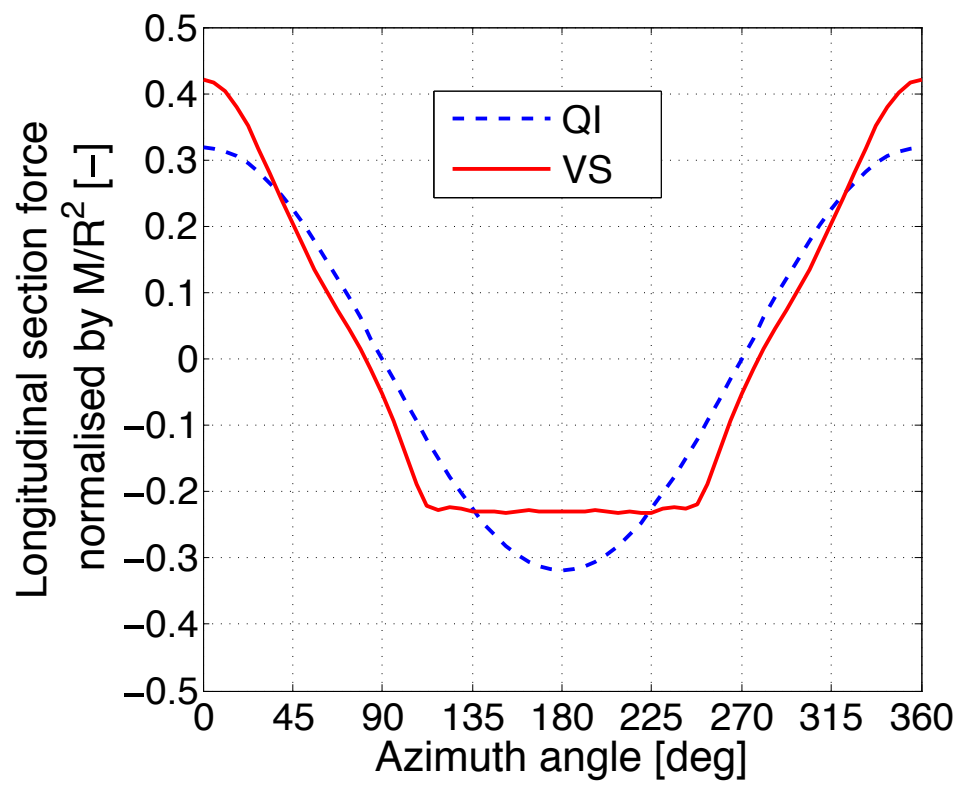

(b) Longitudinal section force

Figure 1. Longitudinal strain and section force distribution around the circumference of circular cylinder under bending 1000 in-lbs for quasi-isotropic (QI) and variable stiffness (VS) design. 


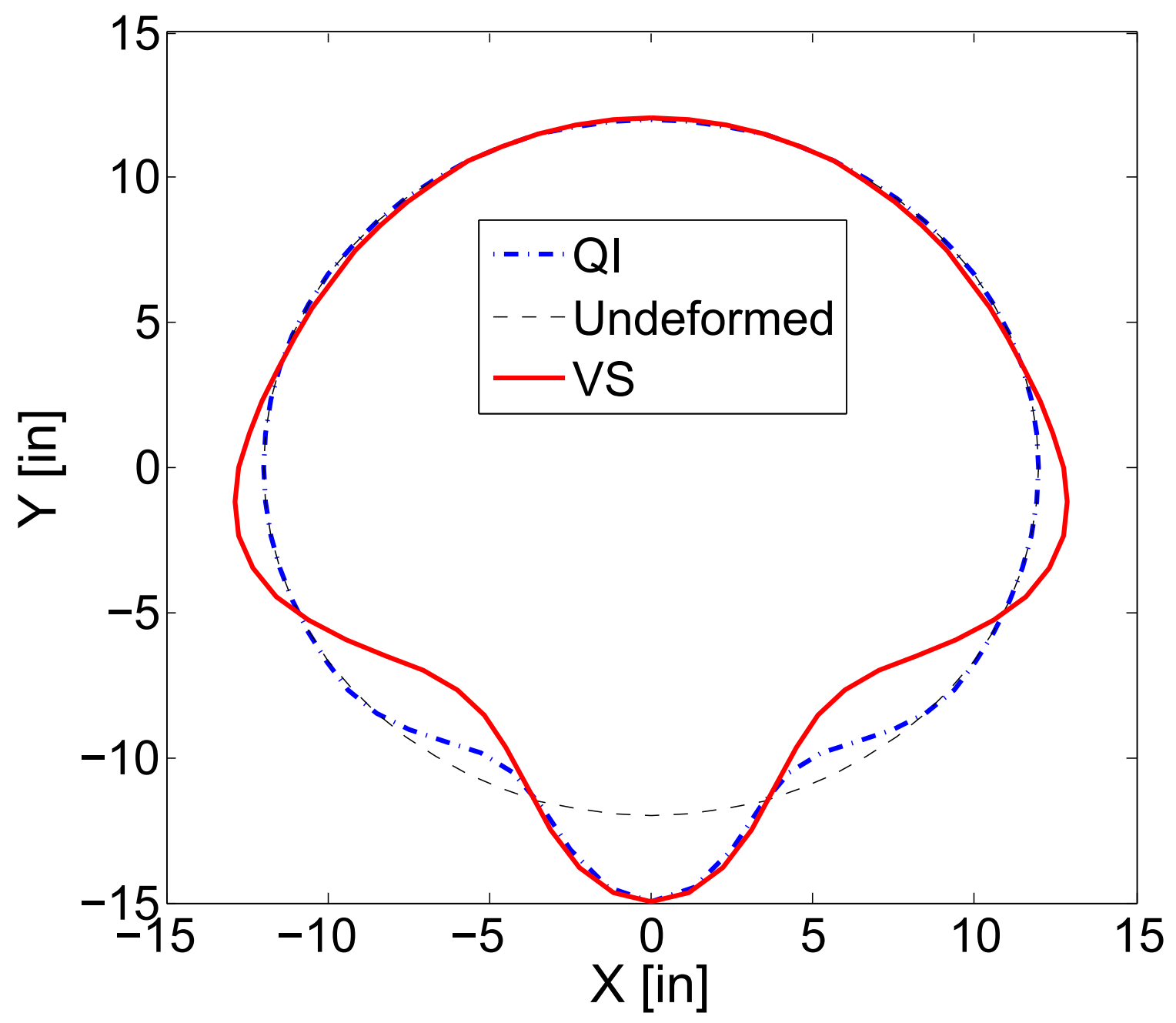

Figure 2. Cross-sectional mode shapes for quasi-isotropic (QI) and variable stiffness (VS) design of the circular cylinder under bending. There are 10 and 1 axial half-waves for the critical mode in QI and VS design respectively 


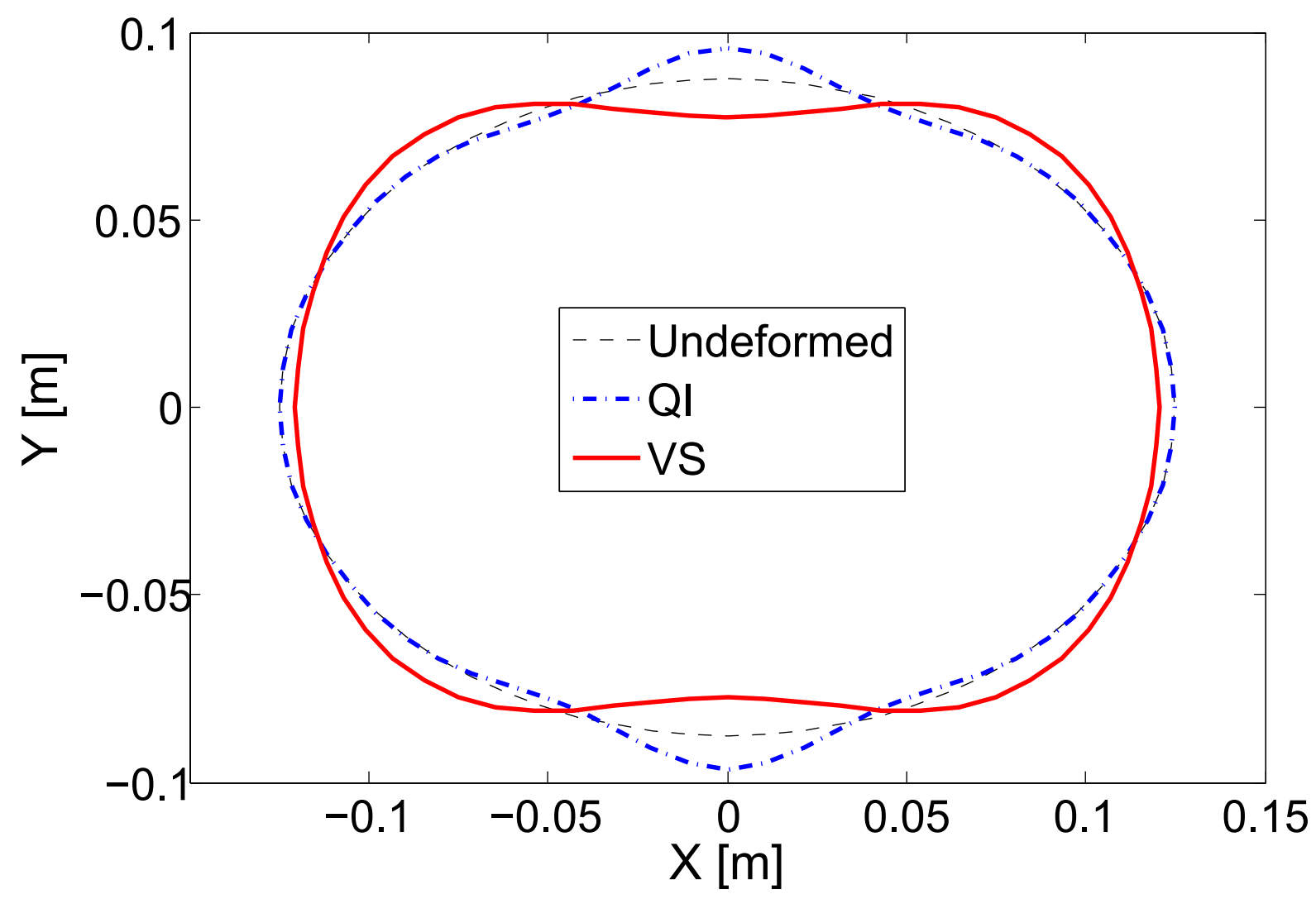

Figure 3. Cross-sectional mode shapes for quasi-isotropic (QI) and variable stiffness (VS) design of the elliptical cylinder under axial compression. There are 10 and 1 axial half-waves for the critical mode in QI and VS design respectively 


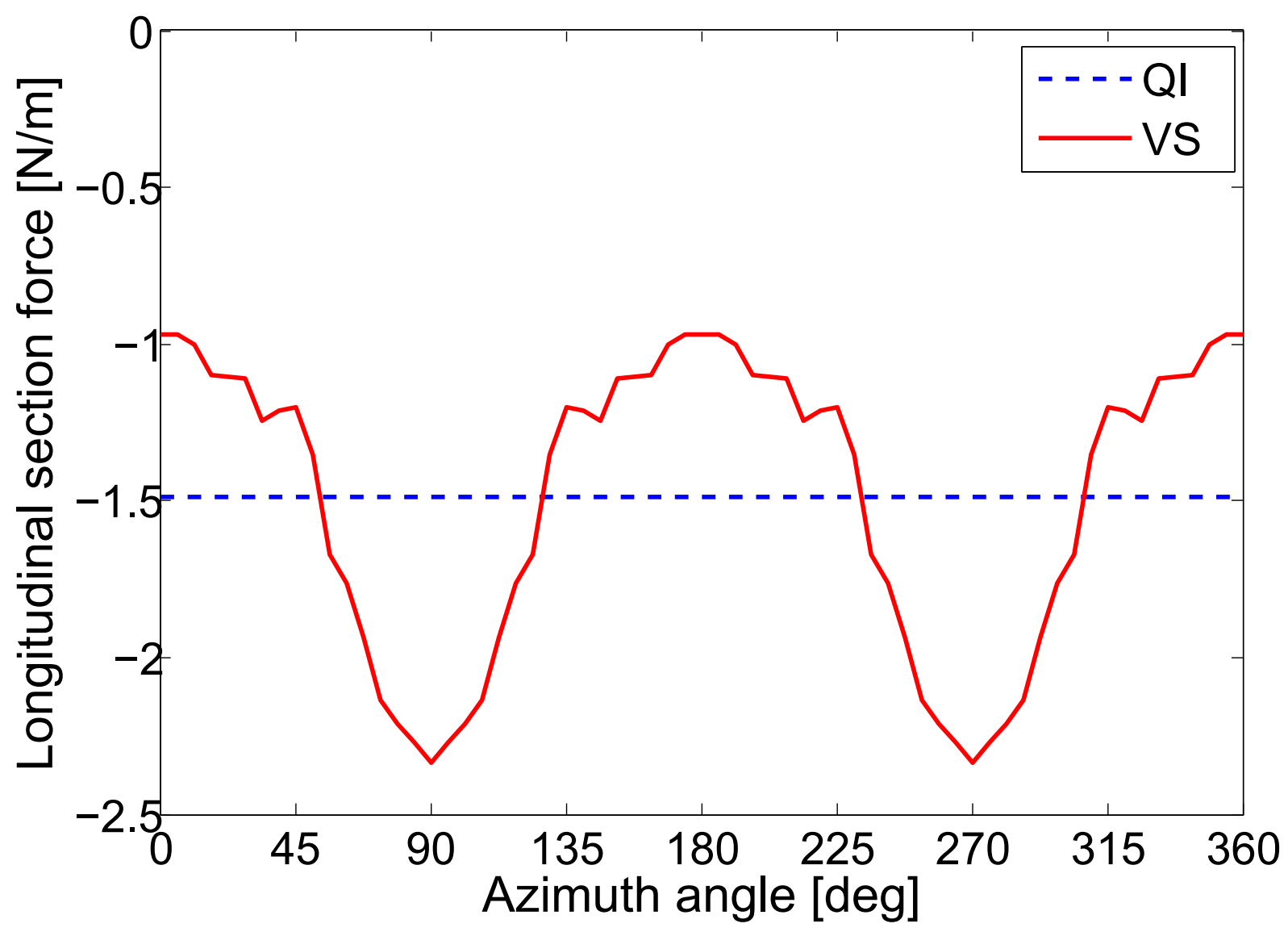

Figure 4. Distribution of longitudinal section force for quasi-isotropic (QI) and variable stiffness (VS) design of the elliptical cylinder under axial compression $1 \mathrm{lbs}$. 


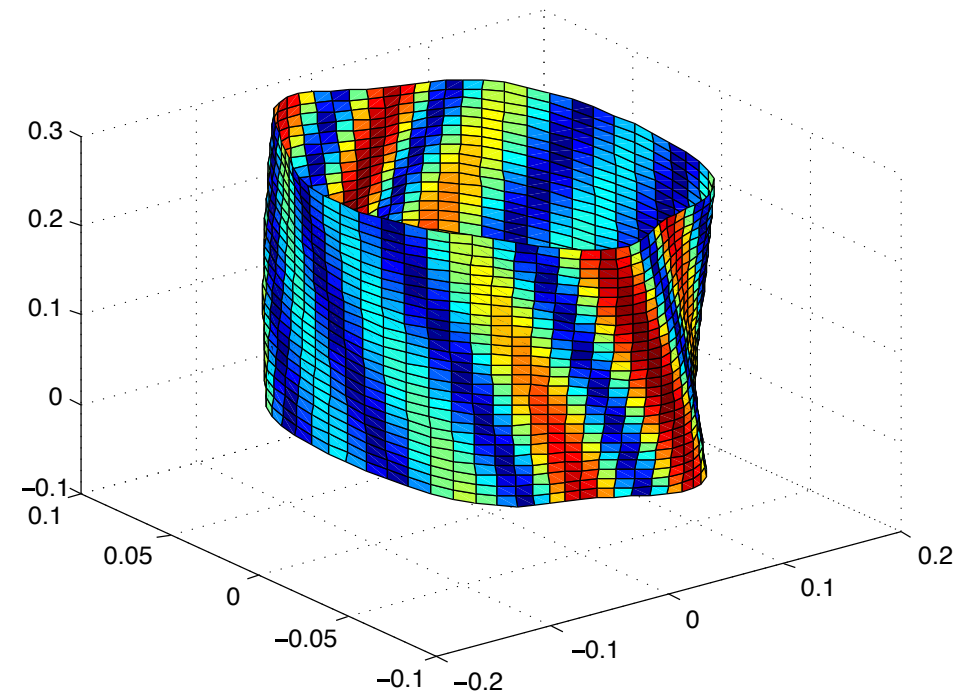

(a) Critical mode shape for quasi-isotropic design

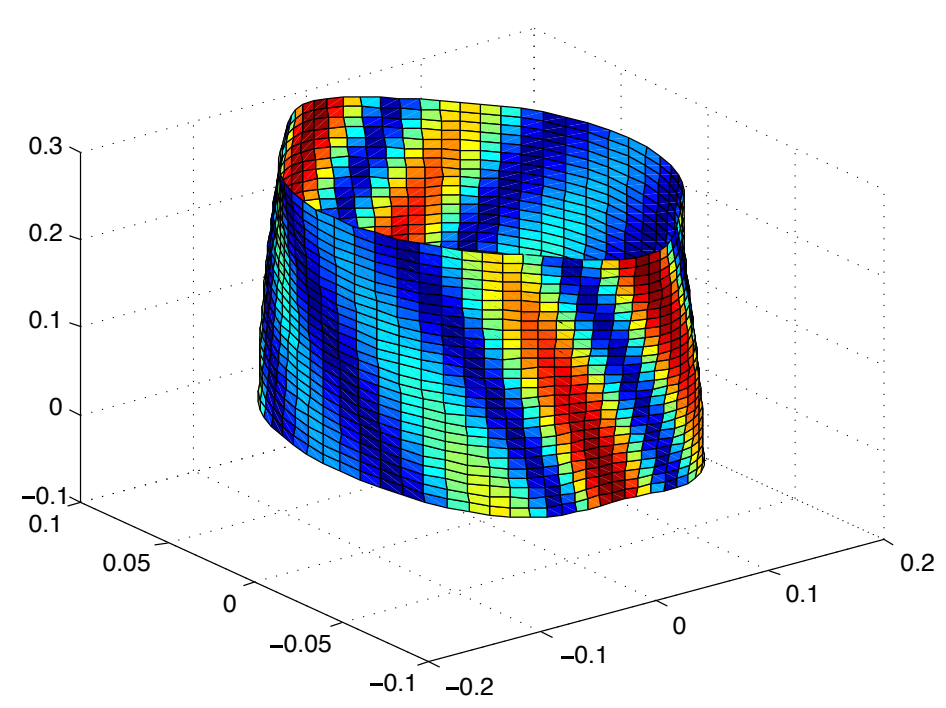

(b) Critical mode shape for variable stiffness design

Figure 5. Critical mode shapes for quasi-isotropic and variable stiffness designs under torsion. 